Meta

Journal des traducteurs

Translators' Journal

\title{
Cognition et déverbalisation
}

\section{Christian Balliu}

Volume 52, numéro 1, mars 2007

Traductologie : une science cognitive

URI : https://id.erudit.org/iderudit/014714ar

DOI : https://doi.org/10.7202/014714ar

Aller au sommaire du numéro

Éditeur(s)

Les Presses de l'Université de Montréal

ISSN

0026-0452 (imprimé)

1492-1421 (numérique)

Découvrir la revue

Citer cet article

Balliu, C. (2007). Cognition et déverbalisation. Meta, 52(1), 3-12.

https://doi.org/10.7202/014714ar

\section{Résumé de l'article}

Le développement actuel des recherches en sciences cognitives permettra d'éclairer nombre de processus à l'oeuvre dans le travail du traducteur et de l'interprète. Cet article étudiera l'attention et la mémoire sous un angle psychologique, dans leur rapport à la déverbalisation. S'interroger sur leur mode de fonctionnement devrait améliorer à terme la pédagogie de la traduction et de l'interprétation.
Ce document est protégé par la loi sur le droit d'auteur. L'utilisation des services d'Érudit (y compris la reproduction) est assujettie à sa politique d'utilisation que vous pouvez consulter en ligne.

https://apropos.erudit.org/fr/usagers/politique-dutilisation/ 


\title{
Cognition et déverbalisation
}

\author{
CHRISTIAN BALLIU
}

ISTI, Haute École de Bruxelles, Belgique et ISIT, Paris, France

cballiu@heb.be

\begin{abstract}
RÉSUMÉ
Le développement actuel des recherches en sciences cognitives permettra d'éclairer nombre de processus à l'œuvre dans le travail du traducteur et de l'interprète. Cet article étudiera l'attention et la mémoire sous un angle psychologique, dans leur rapport à la déverbalisation. S'interroger sur leur mode de fonctionnement devrait améliorer à terme la pédagogie de la traduction et de l'interprétation.
\end{abstract}

\begin{abstract}
The current development of research in cognitive science is sure to shed light on a number of processes involved in the work of the translator and the interpreter. The present paper will study attention and memory from a psychological point of view in their relation to "deverbalization." Probing into their way of working should in the long run improve the teaching of translating and interpreting.

\section{MOTS-CLÉS/KEYWORDS}

cognition, attention, mémoire, déverbalisation, pédagogie de la traduction et de l'interprétation
\end{abstract}

Le titre de cet article, un peu sec, ne reflète qu'une partie des travaux d'Hercule qui se présentent au traducteur lorsqu'il entreprend de traduire un texte. Effectivement, dans la Théorie interprétative de la traduction (théorie du sens), la déverbalisation n'est que la deuxième des trois phases nécessaires à la médiation interlinguistique, alors que la cognition est mobilisée dans l'ensemble du processus. Par ailleurs, la déverbalisation ne concerne bien entendu pas la seule interprétation; le traducteur (entendez celui qui s'occupe de textes écrits) utilise le même processus.

Il est intéressant de constater que la traductologie, discipline jeune en tant qu'activité scientifique et justiciable d'un enseignement universitaire, mais ancienne en tant que dispositif de réflexion, n'avait pas intégré dans sa réflexion jusqu'à il y a peu la dimension cognitive.

La cognition est ce que l'on appellerait volontiers une notion carrefour, tant il est vrai qu'elle se situe à la charnière des cinq grands axes qui gouvernent actuellement les recherches dans le domaine. S'intéressent à la cognition les neurosciences, la psychologie, l'intelligence artificielle, la linguistique et la philosophie, le tout venant à former les sciences cognitives. Gardner préfère évoquer un hexagramme où l'anthropologie vient jouer le sixième larron (1993: 52). L'hexagramme de Le Moigne remplace quant à lui la philosophie par l'épistémologie et l'anthropologie par les sciences sociales (Le Moigne cité par Salanskis 2003: 62). Cette pluridisciplinarité, féconde sur le plan de la production, mais ingrate sur le plan de la coordination scientifique, se heurte d'emblée à deux problèmes cruciaux: la disparité des sujets d'expérimentation et la variété des dispositifs d'interprétation. 
Selon que le sujet d'expérimentation est le cerveau physiologique, l'esprit et sa dimension psychologique, l'ordinateur, le langage comme moyen de matérialisation cognitive ou encore la composante philosophique qui sous-tend la recherche, on ne «parle pas de la même chose» et la pluridisciplinarité ne se mue pas nécessairement en transdisciplinarité. Dans le même ordre d'idées, l'expérimentation et la modélisation proposées différeront en fonction du sujet, vivant, virtuel ou insaisissable s'agissant de psychologie. On pourrait à cet égard s'étonner de ce que psychologie, philosophie et linguistique constituent trois entrées distinctes, alors que la psychologie du langage semble être un partenaire indissociable des études sur la connaissance. Mais la psychologie du langage ne s'est intéressée que fort tard à cette autre discipline qu'est la traduction, alors qu'elle pouvait trouver dans les faits de bilinguisme un terrain d'exploitation particulièrement fertile. Il est vrai au demeurant que les psychologues ne se sont dans un premier temps penchés sur le langage que dans la mesure où il pouvait être le révélateur de certains troubles psychologiques. Charcot et Freud comprirent que le langage est une voie privilégiée d'accès à l'esprit, singulièrement dans le domaine de la psychopathologie.

La jeunesse institutionnelle des sciences cognitives (Pélissier et Tête 1995) explique ces maladies d'enfance et n'est pas sans incidence sur la labilité terminologique qui les caractérise et, par conséquent, sur le statut épistémologique de celles-ci, avec le risque d'un "pluriel de dispersion». En effet, des termes comme apprentissage, attention, perception, représentation et bien d'autres seront utilisés dans un sens différent en fonction de la discipline en question. Le mot interprétation lui-même n'a bien entendu ni la même définition ni la même résonnance chez Seleskovitch et Rastier par exemple (Plassard 2005). C'est ainsi que le vocabulaire utilisé, dont les sens varieront selon les domaines voire les auteurs, sera dans chaque occurrence un acte épistémologiquement fondateur.

La cognition est le mécanisme qui nous permet «de comprendre et d'acquérir des connaissances, ainsi que de les traiter» (Stuart-Hamilton 1995: 20). Ce mécanisme joue un rôle essentiel en interprétation et, bien sûr, dans la déverbalisation. Nous pourrions dire de manière plus familière: comprendre, emmagasiner, régurgiter, et surtout appliquer et utiliser à de nouvelles fins, ce qui nous intéresse en matière de traduction et d'interprétation.

Le sens est compris par le traducteur (ou l'interprète) lorsqu'il ente des compléments cognitifs et affectifs sur un énoncé. La somme du linguistique et de l'extralinguistique confère donc à l'énoncé un sens, ce qui le distingue fondamentalement de la phrase, laquelle est une notion strictement linguistique. C'est-à-dire que le passage des signifiants de la langue source vers les signifiants de la langue cible est court-circuité par ce que Nida appellerait la "phase de transfert», une auscultation médiate du texte qui met les mots entre parenthèses pour n'en retenir que le sens. L'interprétation du sens, au moyen d'un processus inférentiel, permettra de traduire enfin le message. Mais quels sont ces éléments cognitifs? Quels sont les mécanismes qui permettent que la déverbalisation ait lieu dans l'esprit de celui qui traduit?

Les sciences cognitives, et plus précisément la psychologie cognitive, se sont penchées sur l'étude du langage et sur les mécanismes de fonctionnement de l'esprit. Elles en ont identifié un bon nombre, dont je citerai et commenterai ceux qui touchent le plus à l'interprétation, à savoir l'attention et la mémoire. Ces deux mécanismes sont aussi profondément liés aux processus de déverbalisation. Si le mot processus est au 
pluriel, c'est parce qu'il y a à mon sentiment deux types de déverbalisation: le premier fait que le sens apparaît souvent d'emblée, spontanément, indépendamment des signes linguistiques qui le matérialisent ou parfois l'occultent; le second est la déverbalisation telle que décrite par la théorie du sens de Paris, qui est une trace mnésique désambigüisée, débarrassée de sa gangue lexicale.

\section{L'attention}

L'attention est, selon la définition de William James, «la capacité que l'individu possède de prendre possession de son esprit de façon consciente pour focaliser ses ressources cognitives sur une pensée ou un objet particulier parmi plusieurs autres. Cela implique que l'on rejette un certain nombre d'éléments afin de gérer efficacement les autres » (Mialet 1999: 11). Plus simplement, «l'attention est liée à la manière dont le système cognitif traite l'information» (Camus 1996).

Dans les années 1970, Posner, Schneider et Shiffrin ont fait le départ entre les processus attentionnels automatiques, rapides, parallèles et désintéressés, et les processus attentionnels conscients lents, séquentiels et stratégiques, c'est-à-dire obéissant à une fonction de contrôle par le sujet, à une motivation.

Il y a donc, pour le dire autrement, un processus de contrôle et de sélection qui renvoie au modèle de pertinence de Sperber et Wilson. Les psychologues y voient surtout une métaphore exercée sur des représentations mentales et adossée à des processus d'activation et d'inhibition. La notion de bénéfice me paraît dès lors indissociable du processus de sélection.

Pour sa part, Donald Broadbent a avancé l'hypothèse du «filtre attentionnel» une autre métaphore qui trahit une absence patente de contenu -, lequel bloquerait toute information non pertinente (Bowring 2003). Des recherches plus récentes semblent indiquer que le «filtre attentionnel» ne crible pas complètement toutes les informations mais qu'il atténue seulement celles qui sont abandonnées (Mialet 1999: 53). Il peut en conséquence se produire des interférences et des troubles de l'attention.

L'attention pourrait aussi être divisée pour accomplir plusieurs tâches simultanées, sans que les niveaux des performances en soient affectés pour autant (Bowring 2003). C'est ce que met en évidence l'expérience de la «double-tâche» qui permet notamment d'étudier in vivo les mécanismes attentionnels automatiques et les mécanismes attentionnels conscients.

Cela a permis au psychologue américain Alan Allport de proposer un modèle à plusieurs canaux, qui travailleraient en parallèle et seraient à même de traiter séparément chaque parcelle d'information (Allport et al. 1972: 225-235). Suivant ce raisonnement, toute tâche complexe mettrait en jeu plusieurs processeurs en même temps. C'est pour cela qu'une opération multitâche, comme l'interprétation par exemple, est possible dès lors que les différents canaux traitent des opérations distinctes. Il est à remarquer ici que la notion d'entraînement, si importante en matière d'attention, de mémoire et d'interprétation de conférences, est curieusement absente de ces travaux.

Lors d'une interprétation simultanée, l'interprète doit écouter le discours, suivre la proxémique et les intonations de l'orateur, comprendre ce discours, le déverbaliser pour le traduire mentalement, le reformuler dans l'autre langue sans discontinuité, tout en répétant ces opérations inlassablement dans sa course contre la fugacité de 
l'oral. Il doit aussi parfois noter des chiffres, des noms propres, des termes techniques, des énumérations, aux fins conjointes de ne pas perdre de l'information et de poser des choix traductifs pertinents. À ce propos, il n'est pas inutile de rappeler qu'en simultanée, les interprètes sont souvent au nombre de deux par cabine et que celui qui n'a pas le tour de parole en profite pour aider son collègue, en prenant des notes, en faisant éventuellement des recherches terminologiques, en glanant des informations utiles, en soulageant la mémoire de celui qui travaille par des annotations, etc. La combinaison, fréquente dans la pratique de la simultanée, de deux interprètes dans la même cabine me paraît insuffisamment étudiée.

Toutes ces tâches réclament une attention particulièrement soutenue, une grande endurance physique, mentale et nerveuse ainsi que plusieurs efforts distincts et simultanés qui peuvent mener l'interprète à la saturation et lui faire commettre des erreurs.

On comprendra dès lors qu'il est judicieux d'étudier la mémoire de plus près pour tenter d'appréhender au mieux les processus cognitifs qui sont à l'œuvre dans l'esprit de l'interprète.

\section{La mémoire}

La mémoire est le stock de connaissances et d'informations à la disposition du sujet et qu'il convoque par un processus d'activation pour comprendre ou relier des faits, des événements, des données, des énoncés, en vérifier la pertinence et la cohérence et, dans le cas qui nous occupe, traduire ou interpréter. Le traducteur et l'interprète la sollicitent donc en abondance comme un puits de savoir organisé qui peut, à la demande ou automatiquement, émerger à la conscience. On devine déjà que la mémoire jouera un rôle essentiel dans la déverbalisation, exercice difficile s'il en est dans la mesure où nous avons l'habitude, depuis notre plus tendre enfance, d'écouter ou de lire des mots. Le véhicule linguistique a pris une telle importance dans notre quotidien - j'ai coutume de dire que, si les physiciens ont l'apanage de la physique et les médecins celui de la médecine, les linguistes n'ont pas un droit de préemption sur la langue; nous sommes tous des linguistes, nous «sommes tous tombés dans la langue en étant petits» - qu'il en arrive à occulter la possibilité d'une pensée et d'une perception averbales. La madeleine de Proust, dans son économie du signifiant au profit du référent, n'est pas étrangère à la déverbalisation.

La psychologie cognitive s'intéressera davantage au versant linguistique de la mémoire, alors que les neurosciences s'emploieront à définir les mécanismes perceptifs et la nature des représentations dont ces mécanismes font l'objet. Les spécialistes de la psychologie et des neurosciences tentent en conséquence de modéliser l'architecture et le fonctionnement de notre mémoire, afin de comprendre comment les informations sont emmagasinées et comment elles en sont, parfois de nombreuses années plus tard, extraites à la faveur d'une demande (convocation) ou d'une association fortuite (évocation). Les troubles de la mémoire sont également à l'étude aujourd'hui, y compris dans le cadre de la maladie d'Alzheimer, pour identifier l'étiologie, tantôt physiologique, tantôt mécanique, tantôt psychologique, des déficits mnésiques. Les causes sont d'ailleurs souvent multiples.

Ces essais de modélisation obéiront, sur le plan d'un postulat épistémologique, à cette distinction fondamentale entre le cognitivisme, qui fait la part belle aux symboles 
et qui pense le cerveau à l'image de l'ordinateur - « raisonner, c'est calculer ", écrivait Hobbes en 1751 -, et le connexionnisme, qui croit en l'omnipotence de la perception et qui pense l'ordinateur à l'image du cerveau. Quelle que soit la philosophie adoptée, des travaux plus importants en matière de développement cognitif et de métacognition seraient les bienvenus; se posent en effet nombre de questions sur l'objectivité de nos grilles de lecture et de notre introspection puisqu'il y a en tout état de cause autoanalyse du sujet.

La littérature s'accorde à distinguer plusieurs types de mémoire en fonction de sa fugacité et de sa fragilité, d'une part, de sa continuité et de sa stabilité, de l'autre: la mémoire à court terme (dite aussi mémoire de travail) et la mémoire à long terme. Cependant, il ne faut pas perdre de vue que la mémoire, quelle que soit la ventilation qu'on lui impose, se caractérise par un dynamisme chronique et, si la dichotomie établie peut s'avérer pratique et fonctionnelle, elle n'est pas nécessairement pertinente du point de vue opératoire.

Durieux (2006: 22-26) a bien synthétisé la structuration et le fonctionnement de la mémoire qui est, somme toute, un réceptacle de savoirs dont les tiroirs, aussi nombreux et extensibles soient-ils, sont capables de s'ouvrir et de livrer leurs secrets, parfois enfouis depuis de nombreuses années dans le cas de la mémoire à long terme, en une fraction de seconde.

C'est ici que se profile le mystère de la représentation, des images mentales. On ne stocke pas des objets, des senteurs, des livres; on stocke des symboles qu'on structure à sa manière par des procédés mnémotechniques intrinsèques. La représentation peut être visuelle, spatiale, articulatoire ou simplement linguistique. C'est précisément ce paradigme symbolique qui sonne le glas, aux yeux des cognitivistes, du connexionnisme, qui évacue la manipulation des symboles sur l'autel d'un complexe neuronal.

La mémoire à court terme est très sollicitée lors d'une séance de travail, par exemple en traduction et en interprétation, lorsqu'il s'agit de construire le sens au moyen de ce qui a été précédemment formulé. La mémoire à court terme aide le sujet à vérifier la connexité, la cohésion et la cohérence d'un énoncé. Bien entendu, la mémoire n'est pas la seule à «aller au charbon». On peut légitimement reprocher aux psychocogniticiens d'étudier le fonctionnement de la mémoire en dehors d'une approche contextuelle; la mémoire se déploiera plus aisément et plus efficacement dans un contexte de pertinence avec lequel elle collaborera par un processus de va-et-vient constants. La mémoire à long terme est d'ailleurs tout aussi associée à ce processus. Cela me rappelle l'anecdote (tiens? le fonctionnement de la mémoire...) de cet interprète de l'ONU réputé pour traduire les orateurs au départ d'une dizaine de langues et qui expliquait cette performance à un parterre de journalistes médusés par la boutade suivante: "mais que voulez-vous qu'ils disent?» Seleskovitch ne pensait pas à autre chose lorsqu'elle me confiait que ses interprétations les plus délicates avaient été celles où elle ne dominait pas le sujet. Ces considérations sont cruciales pour le travail du traducteur et de l'interprète car elles opèrent un recentrement sur le cogito au détriment d'un travail pseudo-objectif sur le texte (linguistique).

La mémoire à long terme peut être ventilée, selon Schacter et Tulving (1994), en mémoire épisodique et mémoire sémantique. La mémoire épisodique procède par associations, elle situe les informations dans le temps et l'espace, par rapport à des personnes, des événements, des lieux, des coïncidences, et dépend étroitement de faits contextuels pour être sollicitée: c'est l'évocation citée plus haut, c'est un éveil à la 
conscience, au sens où, dans la vie, on ne se réveille pas soi-même, on est réveillé par un bruit, par la sonnerie de son réveil... La mémoire sémantique rassemble les connaissances générales du monde, elle les organise sans tenir compte du moment ni des circonstances de leur constitution, dans le but de faciliter leur récupération: c'est la convocation, liée à une distribution en tiroirs. Cette distribution en fonction de la durée peut être regroupée dans ce qu'il est convenu d'appeler la mémoire déclarative (connaissances matérialisées dans le langage naturel ou par des images mentales).

Anderson (1983) avait, de manière très intéressante, distingué mémoire déclarative et mémoire procédurale, cette dernière intervenant dans la réalisation d'activités perceptives, motrices ou cognitives et étant moins perméable à la conscience ( «on ne désapprend pas à rouler à bicyclette»).

Cordier et Gaonac'h définissent plus simplement la mémoire comme « un dispositif qui permet de construire et de maintenir des connaissances en vue de leur utilisation » (2004: 33) qui fonctionne, d'après les hypothèses des psychologues cognitifs, suivant trois étapes:

- Dans un premier temps, l'information reçue par le cerveau est emmagasinée. La façon dont l'information sera emmagasinée variera en fonction de la méthode utilisée pour la coder. Pour retrouver l'information par la suite, le cerveau sera conditionné par la méthode qu'il aura utilisée pour l'encoder; ce que j'appellerais volontiers le «syndrome du Petit Poucet». Ce sont les variantes de codage qui ont conduit les psychologues à considérer qu'il existe différents types de mémoire;

- Le sujet retient ensuite l'information encodée. Cette étape est d'une durée variable (voilà la raison pour laquelle on parle de mémoire à court ou à long terme). C'est aussi cette étape qui permet de traiter et de transformer l'information en vue de la consolider dans notre esprit. Il serait intéressant, à ce stade, d'affiner les mécanismes qui consolident les informations dans la mémoire et de repérer ceux qui les en effacent. Les fameux «trous de mémoire», momentanés par essence, obéissent aussi à des mystères encore à élucider;

- La dernière étape est celle de l'actualisation ou de la réactualisation, au cours de laquelle l'individu utilise ce qu'il a codé et retenu. Pour ce faire, l'information sera d'abord récupérée «en vrac» et sera criblée en fonction de sa pertinence à produire une réponse.

Dehaene (2001: 9) postule l'existence de plusieurs formes de codage de l'information (visuel, auditif, tactile, etc.) qui donnent lieu à des types de mémoire spécifiques. Seleskovitch a raison quand elle signale que «à toute perception ponctuelle s'associe un schème beaucoup plus vaste, et la résultante de cette association est à la fois compréhension et souvenir. Le détail de la perception, objet ou parole, disparaît; par contre, subsiste le souvenir de ce qui a été compris» (1975: 99). Si les sonorités de la parole disparaissent aussitôt, l'auditeur conserve un souvenir déverbalisé, «un état de conscience de l'idée ou du fait évoqué» selon Lederer (1993: 633). Et d'ajouter: «L'oral disparaît avec ses significations, seuls subsistent des sens dans la mémoire de ceux qui ont été intéressés par le discours. L'écrit survit à ses destinataires premiers et ne présente plus au bout d'un temps que ses significations, qui offrent aux lecteurs ultérieurs une infinité d'interprétations.» On peut donc conclure que notre système de codage est essentiellement contextuel et que ce que l'on mémorise est toujours codé hic et nunc, avec et dans son contexte, et non in abstracto.

Après avoir esquissé le fonctionnement de l'attention et de la mémoire, il convient d'analyser leur rôle dans la déverbalisation. 


\section{La déverbalisation sous l'angle de la psychologie cognitive}

Dans le cadre de son travail de fin d'études mené sous ma direction à l'ISTI, López Martínez (2005:39) a tenté d'expliquer comment s'opère la déverbalisation au niveau psychologique chez un interprète. Certains éléments repris dans le schéma ont déjà été expliqués et définis, mais non traités ensemble.

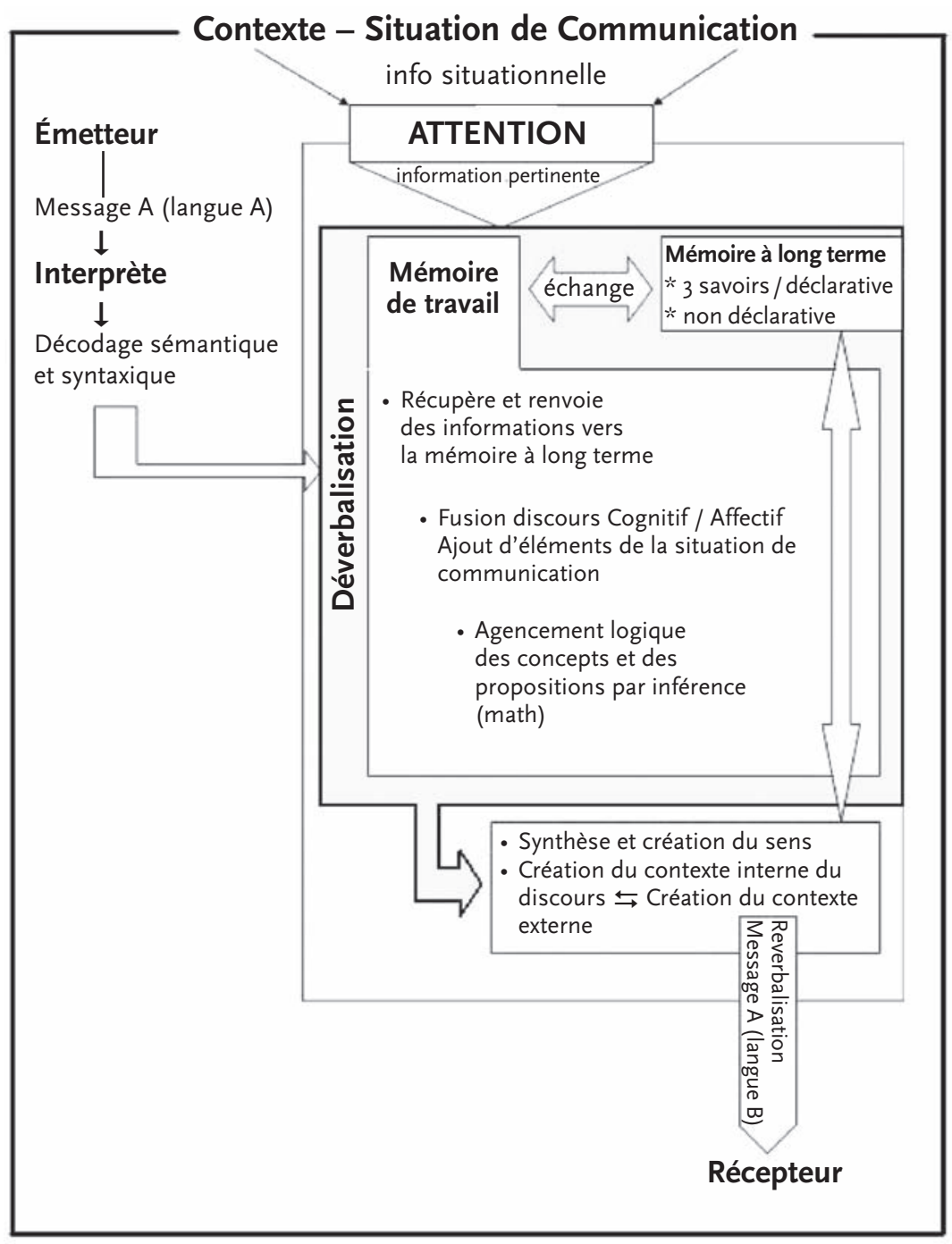

Ce schéma illustre le contexte. Le contexte englobe tous les acteurs qui prennent part à la situation de communication. Il fournit un certain nombre d'informations situationnelles aux acteurs, qui sont au nombre de trois dans le schéma (émetteur, interprète, récepteur). Ceux-ci reçoivent cette information et vont la traiter pour ajouter ses éléments les plus pertinents à l'information contenue dans le discours prononcé par l'orateur. 
L'émetteur du discours va prononcer le message A (en langue A) qui va arriver aux oreilles de l'interprète. Ce dernier fera office de récepteur temporaire du message et va déverbaliser celui-ci, c'est-à-dire le désambigüiser par une alchimie dont - paradoxalement - le bon interprète maîtrise l'application sans en décrypter le processus. Ce qui explique, à défaut de mieux, le foisonnement métaphorique d'un Ladmiral (2005: 478-479) : le «salto mortale», le «no man's langue», le «déjà-plus et le pasencore»... Autrement dit, l'interprète décode les éléments sémantico-syntaxiques qui lui parviennent, il déshabille le texte source, il se défait de l'apparat linguistique. On sait combien ce point retient l'attention des professeurs d'interprétation et de traduction: «le mirage du texte écrit est sans doute l'une des hallucinations les plus tenaces de l'étudiant. Il lui brouille la vue, il engourdit sa clairvoyance» (Balliu 2003: 20).

Les informations que l'interprète aura tirées du discours vont tout d'abord traverser un des processeurs de l'attention. Après leur analyse, les concepts les plus pertinents vont être envoyés vers la mémoire à court terme (ou mémoire de travail) pour y être traités. Simultanément, une série d'informations issues du contexte seront triées par un autre processeur de l'attention et vont être envoyées à leur tour vers la mémoire à court terme.

Les informations pertinentes extraites conjointement du discours et du contexte vont alors être emmagasinées dans les systèmes esclaves de la mémoire à court terme. Le processeur central entame ensuite le traitement de ces informations. Pour cela, il fera appel aux réseaux mnésiques de la mémoire à long terme qui vont relayer vers la mémoire à court terme des informations liées aux notions, aux concepts évoqués par le discours et l'information situationnelle. Ces informations émaneront des savoirs aussi divers que variés que l'interprète aura acquis et accumulés au long de son existence. Toute l'information proposée par la mémoire à long terme ne sera pas pertinente et seule une partie sera utilisable; c'est ici qu'intervient l'inhibition, ce modulateur de l'activation, ce censeur de l'impropriété.

Toute une série d'images conceptuelles et d'idées vont de la sorte venir occuper le processeur central de la mémoire à court terme et vont favoriser la fusion entre le discours et l'information situationnelle, d'une part, et les compléments cognitifs et affectifs ajoutés par l'interprète, d'autre part; cet écheveau autorisera la synthèse du sens par la formulation et la sélection d'hypothèses sur le contexte.

Puisque le sens est produit par la désambiguïsation et la compréhension, la déverbalisation est alors achevée, dans la mesure où elle constitue une condition sine qua non de réalisation de ce processus. Pour que le sens se rematérialise sous une forme linguistique, il devra être reverbalisé, ce qui correspond à la troisième phase de la théorie du sens de Paris.

Dès lors que la reverbalisation (ou reformulation, réexpression) aura été accomplie, la synthèse du sens et les hypothèses sur le contexte interne (discours) et le contexte externe (global) seront transférées vers la mémoire à long terme pour être emmagasinées.

Ce retour d'information se fera par le biais de la mémoire à court terme, qui retiendra les informations les plus pertinentes pour assurer l'isotopie, à savoir la continuité de la compréhension.

Ce schéma illustre l'importance et les enjeux de l'attention et de la mémoire dans le travail de l'interprète mais aussi du traducteur, et montre qu'il s'agit là de deux 
processus cognitifs majeurs en lice. D'autres processus cognitifs non moins importants entrent également en ligne de compte; ils mériteraient sans conteste un long développement, mais sortent du cadre de la présente étude.

\section{RÉFÉRENCES}

Allport, A., Antonis, B. and P. Reynolds (1972): "On the division of attention: a disproof of the single channel hypothesis", Quarterly Journal of Experimental Psychology 24, p. 225-235.

Anderson, J. R. (1983): The Architecture of Cognition, Cambridge, Harvard University Press.

BAdDeley, A. D. (1993) : La mémoire humaine. Théorie et pratique, Grenoble, Presses Universitaires de Grenoble.

Baddeley, A. D. and L. Weiskrantz (1993): Attention: Selection, Awareness and Control, Oxford, Clarendon Press.

Balliu, Chr. (2003): Louis Leboucher dit Georges Mounin. Textes inédits rassemblés et publiés par Christian Balliu, Bruxelles, Les Éditions du Hazard.

Bowring, T.-S. (2003): The Cognitive Processes Underlying Simultaneous Interpreting: A Review, non publié.

Broadbent, D. E. (1958): Attention and Communication, New York, Pergamon Press.

Camus, J.-F. (1996): La psychologie cognitive de l'attention, Paris, Armand Colin.

Cordier, F. et D. GaOnac'H (2004): Apprentissage et mémoire, Poitiers, Nathan Université.

Damasio, A. (1995): L'erreur de Descartes. La raison des émotions, Paris, Odile Jacob.

Dancette, J. et S. Halimi (2005): «La représentation des connaissances; son apport à l'étude du processus de traduction", Processus et cheminements en traduction et interprétation, Meta 50-2, Presses de l'Université de Montréal, p. 548-559.

Dehaene, S. (2001): The Cognitive Neuroscience of Consciousness, Cambridge, MIT Press.

Durieux, Chr. (2006) : «Complexité et cognition: un paradigme pour la traductologie», Bruxelles, Équivalences 32-2, p. 5-29.

Ehrlich, M.-F., TARdieu, H. et M. Cavazza (1993): Les modèles mentaux-approche cognitive des représentations, Paris, Masson.

GARDNeR, H. (1993): Histoire de la révolution cognitive. La nouvelle science de l'esprit, traduit de l'américain par J.-L. Peytavin, Paris, Payot.

Gaonac'h, D et P. Larigauderie (2000): Mémoire et fonctionnement cognitif: la mémoire de travail, Paris, Armand Colin.

Hirst, W, Spelke, E., Reaves, C., Caharacj, G. and U. Neisser (1980): "Dividing attention without alternation or automaticity", Journal of Experimental Psychology 109, p. 98-117.

Houdé, O, Kayser, D., Proust, J. et F. Rastier (1998): Vocabulaire de sciences cognitives, Paris, Presses Universitaires de France.

LADMiRAL, J.-R. (2002): Traduire: théorèmes pour la traduction, Paris, Gallimard.

Ladmiral, J.-R. (2005): «Le salto mortale de la déverbalisation», Processus et cheminements en traduction et interprétation, Meta 50-2, Presses de l'Université de Montréal, p. 473-487.

LEDERER, M. (1993): «Fondements de la théorie interprétative de la traduction», La traduction au cour de la communication, Actes du XIII ${ }^{e}$ Congrès de la FIT 1, Brighton, Londres, ITI Press, p. 632-641.

LEVINSON, S. (1983): Pragmatics, Cambridge, Cambridge University Press.

López Martínez, M. (2005): Réflexions sur la déverbalisation, mémoire de fin d'études présenté à l'ISTI (Bruxelles) sous la direction de Chr. Balliu, non publié.

Mialet, J-P. (1999): L'attention, Paris, Presses Universitaires de France.

PÉLissier, A. et A. TÊTe (1995): Sciences cognitives. Textes fondateurs, 1943-1950, Paris, Presses Universitaires de France.

Plassard, F. (2005): «Sens ou représentation? Étude comparative de la compréhension selon les modèles cognitifs de la lecture et le modèle interprétatif de la traduction», La théorie interprétative de la traduction, Paris-Caen, Lettres Modernes Minard, t. II, p. 99-133. 
Rastier, F. (1996): Sémantique interprétative, Paris, Presses Universitaires de France.

Roux-Faucard, G. (2005): "La notion d'interprétation chez Rastier et Seleskovitch», La théorie interprétative de la traduction, Paris-Caen, Lettres Modernes Minard, t. II, p. 61-82.

Salanskis, J.-M. (2003): Herméneutique et cognition, Lille, Presses universitaires du Septentrion. Schacter, D. L. et E. Tulving (1994): Memory Systems, Cambridge, MA, The MIT Press.

Seleskovitch, D. (1975): Langue, langage et mémoire, Paris, Lettres Modernes Minard.

Seleskovitch, D. et M. Lederer (2001): Interpréter pour traduire, Paris, Didier Érudition.

Sperber, D. et D. Wilson (1989): La pertinence. Communication et cognition, traduit par A. Gerschenfeld et D. Sperber, Paris, Les Éditions de Minuit.

Stuart-Hamilton, I. (1995): Dictionary of Cognitive Psychology, London, Jessica Kingsley Publishers.

Underwood, G. (1976): Attention and Memory, New York, Pergamon Press. 